\title{
Enhanced Route Selection for Energy Efficiency in Wireless Sensor Networks
}

\author{
Dimitrios J. Vergados \\ Nat. Technical University of Athens \\ School of Electrical and Computer \\ Engineering \\ Iroon Polytexneiou 7, Zografou \\ Athens, GR-157 73, Greece \\ +302107721447 \\ djvergad@telecom.ntua.gr
}

\author{
Nikolaos A. Pantazis \\ University of the Aegean \\ Department of Information and \\ Communication Systems Engineering \\ Karlovassi, Samos \\ GR-832 00, Greece \\ $+302273082220$ \\ npantazis@aegean.gr
}

\author{
Dimitrios D. Vergados \\ University of the Aegean \\ Department of Information and \\ Communication Systems Engineering \\ Karlovassi, Samos \\ GR-832 00, Greece \\ $+302273082220$ \\ vergados@aegean.gr
}

\begin{abstract}
Wireless Sensor Networks (WSNs) facilitate monitoring and controlling of physical environments from remote locations with the best possible accuracy. Sensor networks are dense wireless networks consisting of groups of small, inexpensive nodes, which collect and disseminate critical data. Thus, sensor nodes have various energy and computational constraints, due to their inexpensive nature and ad hoc method of deployment. Considerable research has been focused at overcoming these deficiencies through low-energy consumption schemes. Among, other factors, the route selection strategy may have an impact on the network lifetime. In this paper, we present a lifetime prolonging route selection strategy for wireless sensor networks, namely Low Cost Min-Max Energy Routing (LCMMER). The proposed algorithm tries to avoid the least-energy nodes, while at the same time to maintain low energy consumption for each transmission. Simulation results confirm the superiority of the proposed strategy in terms of node lifetime and network connectivity in comparison with the Minimum Total Transmission Power Routing (MTPR) and the Min-Max Battery Cost Routing (MMBCR) route selection strategies.
\end{abstract}

\section{Keywords}

Wireless sensor networks, energy-efficient routing techniques, shortest path, low-energy sensor nodes

\section{INTRODUCTION}

Wireless Sensor Networks (WSNs) are used more and more in the daily life during the recent years. Medical, environmental and military sectors are some of the most important areas that the recent developments have been applied in. In order to guarantee the wireless sensor networks survivability and increase network lifetime in such special purpose environments, various energy efficiency schemes have been proposed in the literature. In some cases, sensor networks are expected to be able to operate for a long period of time in standby, and transmit the gathered data when required, as soon as possible.

Permission to make digital or hard copies of all or part of this work for personal or classroom use is granted without fee provided that copies are not made or distributed for profit or commercial advantage and that copies bear this notice and the full citation on the first page. To copy otherwise, to republish, to post on servers or to redistribute to lists, requires prior specific permission and/or a fee. MOBIMEDIA 2007, August 27-29, Nafpaktos, Greece

Copyright () 2007 ICST 978-963-06-2670-5

DOI 10.4108/ICST.MOBIMEDIA2007.1856
Energy is a valuable commodity in wireless networks due to the limited battery of the portable devices. The energy conservation problem becomes harder in ad-hoc wireless sensor networks due to their limitations arising from their nature.

One of the factors, that affect the energy consumption of every node in the network, is the policy that is used to select the route between two nodes. This is caused mainly due to two reasons: 1) The energy consumed for every packet transmission is different according to the selection of the routing path. Therefore, a packet that is re-transmitted many times before reaching its destination will use more energy that a packet re-transmitted fewer times. 2) If a route selection strategy uses some nodes consistently more than others, then their energy will be consumed faster, and eventually their energy will deplete. When a critical number of nodes in the network have no energy, then the network will become partitioned, and the communication among the sensors will become impossible. To overcome this problem, power-aware route selection mechanisms are needed. In this paper, a lifetime prolonging route selection strategy for WSNs is proposed. The novelty of this scheme is that it takes into consideration not only the residual energy of each node but also the transmission cost for this route.

This paper is organized as follows: Section 2 discusses the routing protocols and the factors that affect the communication in WSNs. Section 3 presents existing route selection strategies, as well as the proposed strategy. Following, section 4 discuses the network model that is used while section 5 presents the performance evaluation results of the proposed algorithm. Finally, section 6 concludes the research work.

\section{ROUTING TECHNIQUES AND PROTOCOLS IN WSNs}

Due to their common characteristics with ad-hoc networks several routing protocols that were firstly developed for wireless ad-hoc networks are also suitable for wireless sensor networks. These routing protocols may be classified as Flat, Hierarchical and Location-based [3]. We focus our interest on Flat routing protocols for ad hoc wireless sensor networks. These routing protocols can be further classified, according to the routing strategy, in three main different categories: Pro-active (or Tabledriven) protocols, Re-active (or Demand-driven or Sourceinitiated) protocols and Hybrid protocols (Fig. 1) [1]. 


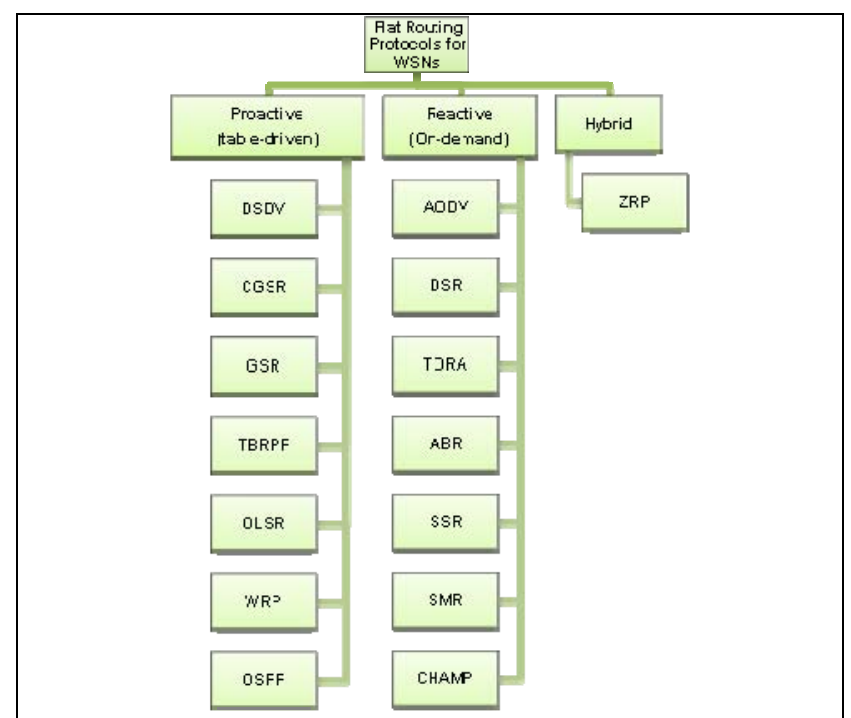

Fig. 1 Flat Routing Protocols Classification for WSNs

Pro-active (or table-driven) routing protocols, such as Destination-Sequenced Distance-Vector Routing Protocol (DSDV) [7], Optimized Link State Routing (OLSR) Protocol. OLSR ([11], [12]) and Open Shortest Path First (OSPF) Protocol [13], routing protocols collect information in advance such that it will be available when need arises. Therefore, each node maintains a full routing table for all destinations and routing updates are used in order to maintain up-to-date information.

Unlike pro-active (or table-driven) routing protocols, re-active protocols (or on-demand protocols) look for information only when needed. For example, when a node needs to reach another node, routes are dynamically created as a result. Some wellknown on-demand ad-hoc routing protocols are: Dynamic Source Routing (DSR) Protocol [8], Temporally Ordered Routing Algorithm (TORA) [9], Ad Hoc On-Demand Distance Vector Routing Protocol (AODV) [10].

Hybrid protocols, like Zone Routing Protocol (ZRP) [14], combine the advantages of both pro-active and re-active routing protocols; some of the nodes may implement a proactive routing protocol and others a reactive routing protocol.

\section{ENERGY-EFFICIENT ROUTE SELECTION POLICIES}

The usual way of routing in ad-hoc wireless networks is to route packets on the minimum-cost path from the source to the destination (sink or base station). The minimum-cost shortest path tree (routed at the base station) connecting all nodes in the network, can be constructed in such a way as to identify the minimum-cost paths from sensor nodes to the base stations. The routing of the data packets to the base station on these minimumcost paths is efficient, so long as the rate of information generation is low or the channel bandwidth is sufficiently high. Still, if the nodes generate data constantly and the bandwidth is constrained, then routing data on the minimum-cost paths can overload wireless links close to the base station [6]. Therefore, a routing protocol must take into consideration the wireless channel bandwidth limitations, otherwise, it might route the packets over highly-congested links and paths. This will lead to increase in congestion, increased delay and packet losses, which in turn will cause retransmission of packets, thereby, increasing energy consumption [6].

Since energy efficiency is a critical issue in wireless sensor networks, existing routing ad-hoc protocols may not be quite suitable for WSNs. Singh et al. [15] proposed several poweraware metrics for determining the routes in wireless ad-hoc networks. More specifically, the authors showed that instead of using hop-count and delay as metrics for route selection for efficient energy routing, it is better to use cost/packet and maximum node cost (which are functions of remaining battery power).

Several route-selection algorithms, that use residual energy transmission power or link distance as the metrics to select an optimal path, may be found into the literature. In this section we present some route selection schemes that try to achieve these goals.

\subsection{The Minimum Total Transmission Power- Routing (MTPR) Algorithm}

The Minimum Total Transmission Power Routing (MTPR) [16] selects the route that uses the minimum total energy consumed in transmission along the route. More specifically, based on the algorithm for each route $l$ the total energy consumed over the route $P_{l}$ is computed:

$$
P_{l}=\sum_{i=0}^{D-1} P\left(n_{i}, n_{i+1}\right)
$$

where $n_{0}$, and $n_{D}$ are the source and the destination nodes respectively, while $P\left(\mathrm{n}_{\mathrm{i}}, \mathrm{n}_{\mathrm{j}}\right)$ denotes the transmission power between two nodes. The selected route $P_{k}$ is the one that satisfies the following property:

$$
P_{k}=\min \left\{P_{l}: l \in A\right\}
$$

where $A$ is the set of all the possible routes.

MTPR may achieve the total power consumption of the overall network. However, since it does not take into consideration the residual energy of each node, it fails to prolong the lifetime of each host [17].

\subsection{The Minimum Battery Cost Routing (MBCR) Algorithm}

The Minimum Battery Cost Routing (MBCR) algorithm [15] selects the route that minimizes the battery cost function. More specifically, for each node it assigns a battery cost function that is given by:

$$
f\left(n_{i}\right)=\frac{1}{c\left(n_{i}\right)}
$$

where $c\left(n_{i}\right)$ denotes the residual energy of node $n_{i}$.

Therefore, the battery cost for a route $l$, length $D$, is given by:

$$
P_{l}=\sum_{i=0}^{D-1} f\left(n_{i}\right)
$$

The selected route $P_{k}$ is the one that satisfies the following property:

$$
P_{k}=\min \left\{P_{l}: l \in A\right\}
$$

where $A$ is the set of all the possible routes. 
The main disadvantage of the MBCR is that the selection is based only on the battery cost, this will lead to increased fairness among nodes, since one node may be overused [2].

\subsection{The Min-Max Battery Cost Routing (MMBCR) Algorithm}

The Min-Max Battery Cost Routing (MMBCR) algorithm [15] selects the route with the maximum values of the minimum residual energies of the nodes.

Therefore, the equation (4) is modified to:

$$
P_{l}=\max _{i \in \text { route } ~} f\left(n_{i}\right)
$$

The selected route $\mathrm{P}_{\mathrm{k}}$ is the one that satisfies the following property:

$$
P_{k}=\min \left\{P_{l}: l \in A\right\}
$$

where $A$ is the set of all the possible routes.

The main disadvantage of the MMCBCR is that it does not guarantee that the total transmission power per packet is minimized over a chosen route [17].

\subsection{Low Cost Min-Max Energy Routing (LCMMER) Algorithm}

Even though the MMBCR strategy prevents the lowest-energy nodes from forwarding packets, and from further reducing their energy, it presents the disadvantage of not considering the cost of the path used when transmitting the information. This strategy may lead to excessively long paths, which will consume large amounts of energy. The Low Cost Min-Max Energy Routing (LCMMER) strategy, proposed in this paper, tries to avoid the least-energy nodes, while at the same maintaining low energy consumption for each transmission. This is realized by applying the MMBCR strategy for all paths that have the minimum cost. That is, from all the admissible paths returned by the routing algorithm, the LCMMER selection strategy filters all the paths with delay larger than a minimum, and keeps all paths that have the minimum possible cost. From these paths, the path containing the least-energy node, with the most energy is selected. Thus, the proposed route selection scheme avoids the least-energy nodes, while at the same time maintaining the cost of the path low, ensuring limited power consumption for each transmission.

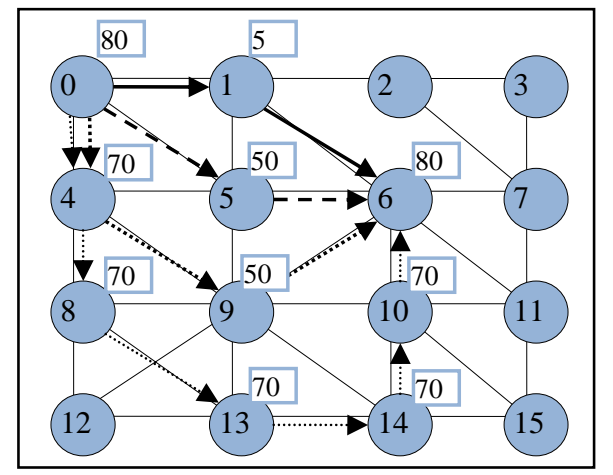

Fig. 2. An example of the LCMMER selection strategy.

The numbers in blue rectangles represent the remaining energy in each node. The arrows represent the possible paths from node 0 to node 6 (redundant paths are omitted). The MTPR strategy would choose the solid path $\{0,1,6\}$, causing a possible depletion of node 5 . The MMBCR strategy would choose the densely dotted path $\{0,4,8,13,14,10,6\}$, causing severe energy waists. The "LCMMER" strategy would choose the dashed path $\{0,5,6\}$, that waists the least energy, and at the same time preserves the network connectivity.

\section{NETWORK MODEL}

The network model that is taken into consideration consists of an ad-hoc sensor network $N$ that contains a number of nodes $n_{i} \in N$. All the nodes in the network use a single wireless channel. The nodes located at a distance shorter than the range are considered neighbors, meaning that they can directly exchange information.

When a node needs to transmit information to another node, a connection (or flow) is generated. When this happens, the routing schemes try to find a path in order to connect the source with the destination node, through a limited number of intermediate forwarding nodes. If an admissible path exists, then the proposed route selection strategy is used for selecting which path will be selected for transmitting the information.

An admissible path may not be found if:

- $\quad$ Either the source or the destination (or both) has depleted its energy.

- There is no path due to network partitioning.

- The only paths that exist are not admissible due to excessive delay.

When no admissible paths are found, then the connection is dropped. When a connection is admitted, the remaining power at each node that participates in the new path is recalculated.

\subsection{Power consumption model}

Since this paper studies the effect of route selection policies on the network lifetime, only the power consumed by the routed information transmissions and receptions is modeled. Thus, nodes are considered to consume energy when they transmit and receive information. Secondary phenomena, like idle listening, collisions, changing states, etc. have been ignored, because they are considered irrelevant to the specific route selection strategy. Also, all packet transmissions are performed at the same power level, regardless of the distance among the sender and the receiver.

For node $n_{i} \in N, P_{i}^{t}$ is the transmitting power consumption, and $P_{i}^{r}$ is the receiving power consumption. Assuming a constant transmission rate $R$, transmitting a message that contains $B$ bits, would cause the source of the message $n_{s}$ to reduce its remaining energy by $\Delta E_{s}=\frac{P_{s}^{t} B}{R}$. Accordingly, the reception of $B$ bits by the destination node $n_{d}$ would reduce its energy by $\Delta E_{d}=\frac{P_{d}^{r} B}{R}$. An intermediate forwarding node 
$n_{f}$ must first receive, and then retransmit the message. Thus,

$\Delta E_{f}=\frac{P_{f}^{t} B}{R}+\frac{P_{f}^{r} B}{R}$.

All nodes are considered to start with the same energy levels.

\subsection{Connection Generation Model}

A random connection generation model was used for the generation of the desired information production. Each connection consists of a directed pair $C_{i}=\left[s_{i}, d_{i}\right]$, where $s_{i}, d_{i} \in N$ are chosen randomly, following a uniform distribution over all the nodes in the network. The connection generator produces a sequence $C_{i}=1, \ldots, C_{\max }$, where $C_{\max }$ is a value chosen large enough, to ensure that the network will be depleted under any route selection algorithm.

\subsection{Performance Metrics}

The basic performance metrics used in the simulation model consist of the number of connections attempted, the number of successful connections, the number of non-depleted (living) nodes in the network, and the connectivity factor of the network.

- Attempted connections: As each simulation round evolves, connections are gradually added to the network. This metric counts the number of connections that are attempted, counting both successful and failed connections.

- Successful Connections: This metric counts only the connections that are successfully admitted in the network, and not the ones that failed. In the beginning of the simulation the successful connections are equal to the attempted ones, since there are no failures. However, as the simulation evolves, the nodes start dying, and the network becomes partitioned, an increasing percentage of the attempted connections fail. Thus, the successful connections metric remains lower than the attempted connections.

- $\quad$ The number of living nodes: This metric is initially equal to 15. As soon as some nodes deplete their energy, this number decreases. A larger number of living nodes is desirable, at all times.

- $\quad$ The connectivity of the network: This metric is considered the most important factor, related to the network lifetime. We define the connectivity factor, at the percentage of the attempted connections that are successful. Due to the uniformly distributed connection generation model, this factor is closely related to the connectivity of the network.

\subsection{Model limitations}

Finally, it should be noted that in this research work node mobility is not taken into consideration. In addition, the actual communication protocol, which is used for creating the topology and for propagating the exchanging of the routing information at each node, is out of the scope of the paper, and the communication overhead is not studied.

\section{SIMULATION RESULTS}

A simulation tool was created for the evaluation of the effectiveness of the MTPR, MMBCR, and LCMMER schemes.
The network topology that was used for the simulation is depicted in Fig. 3.

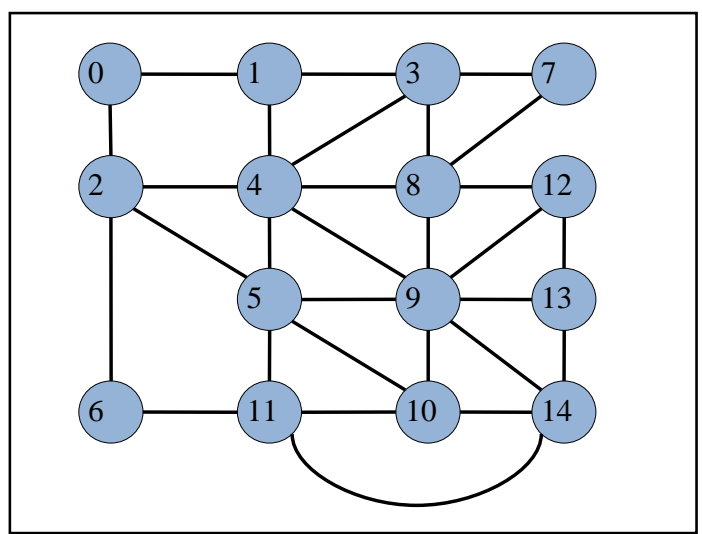

Fig. 3. The wireless sensor network topology

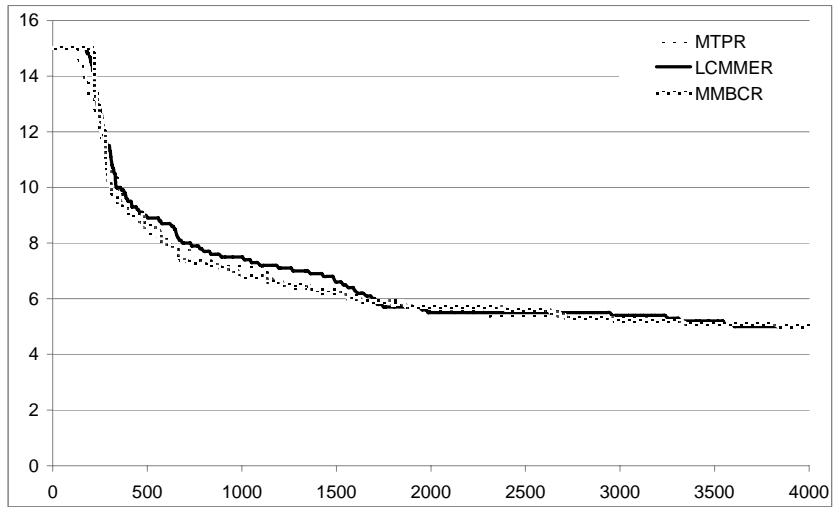

Fig. 4. The number of living nodes as a function of the attempted connections

Fig. 4 illustrates the average number of living nodes, as a function of the attempted connections. The MTPR scheme has the fewest living nodes at all times. In the MMBCR scheme, the first node that dies has a longer lifetime compared to the other two schemes. However, the LCMMER scheme, which always outperforms the MTPR scheme, quickly surpasses the MMBCR scheme also, and retains more living nodes throughout the simulation.

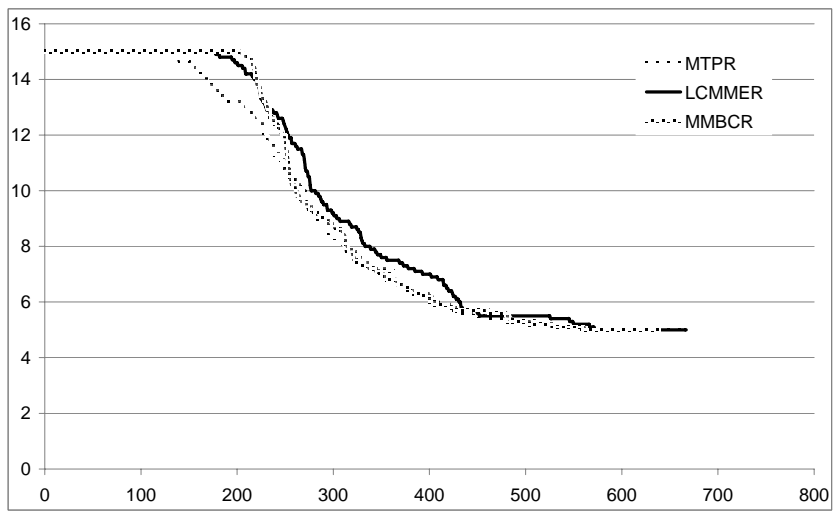

Fig. 5. The number of living nodes as a function of the successful connections

On this topology, the connection generation model described in the previous section was applied, for the three different route 
section strategies under consideration. For each route selection strategy, 10 independent simulation runs were performed, and the results were averaged.

However, Fig 4 shows the performance of the three schemes artificially close together. The main reason for this is the fact that the increased number of the active nodes in the LCMMER causes the success ratio of the network to be higher. Since more connections are successful, more energy in consumed, and thus the lifetime is reduced. This effect is corrected in Fig. 5, which illustrates the average number of living nodes, as a function of the successful connections. In this figure, the advantage of the LCMMER is more obvious, since only the successful connections are taken into consideration.

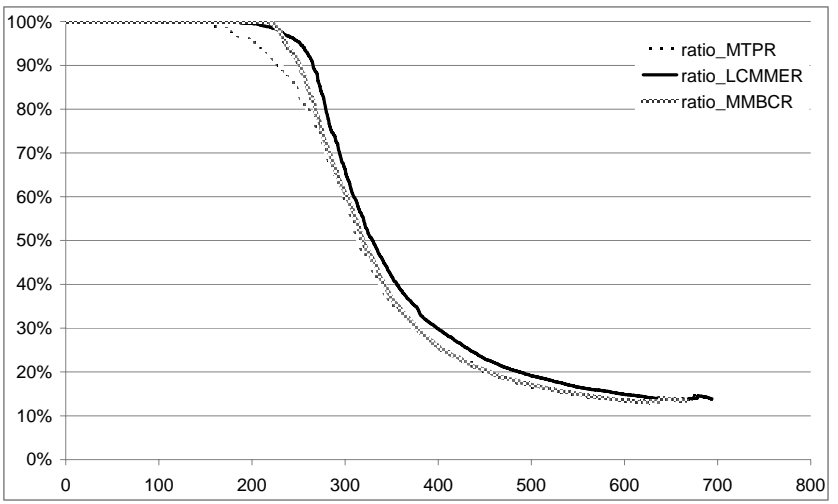

Fig. 6. The connectivity of the network as a function of the successful connections

Fig 6 shows the connectivity of the network for the three route selection strategies. Even though the connectivity of the MMBCR strategy is initially better, the LCMMER scheme becomes more efficient. For the most part of the simulations, the LCMMER strategy achieved steadily a higher connectivity factor.

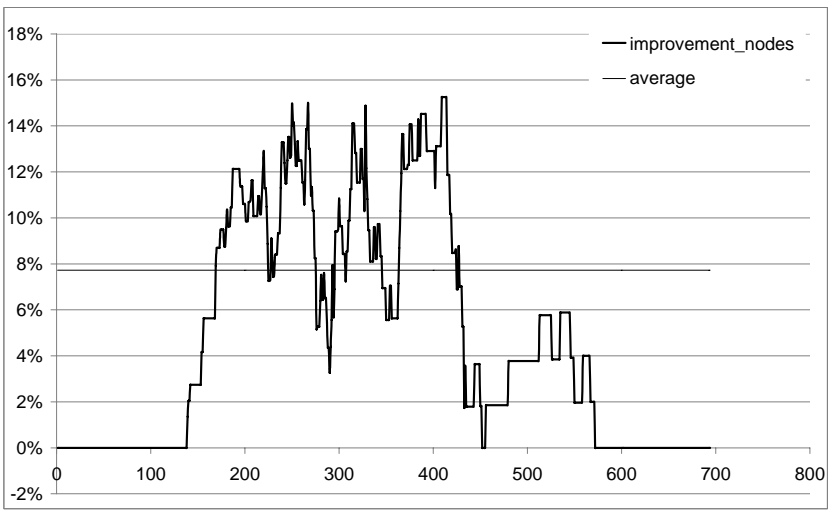

Fig. 7. The improvement of LCMMER compared to the MTPR, in terms of the number of living nodes.

Fig. 7 and Fig 9 show the relative advantage of LCMMER compared to MTPR and MMBCR respectively, in terms of the number of living nodes. The comparative advantage of the proposed scheme is evident, since it is steadily better than the other two schemes, and achieves an on average improvement of $8 \%$ and $6 \%$ respectively. On the other hand, Fig. 8 and Fig. 10 show the relative advantage of LCMMER compared to MTPR and MMBCR respectively, in terms of network connectivity.
From the figures it can be seen that the proposed scheme is steadily better than the other two schemes, and achieves an improvement of on average $11 \%$ and $9 \%$ respectively. Thus the proposed scheme outperforms the other two schemes, both in terms of node lifetime, and in terms of network connectivity.

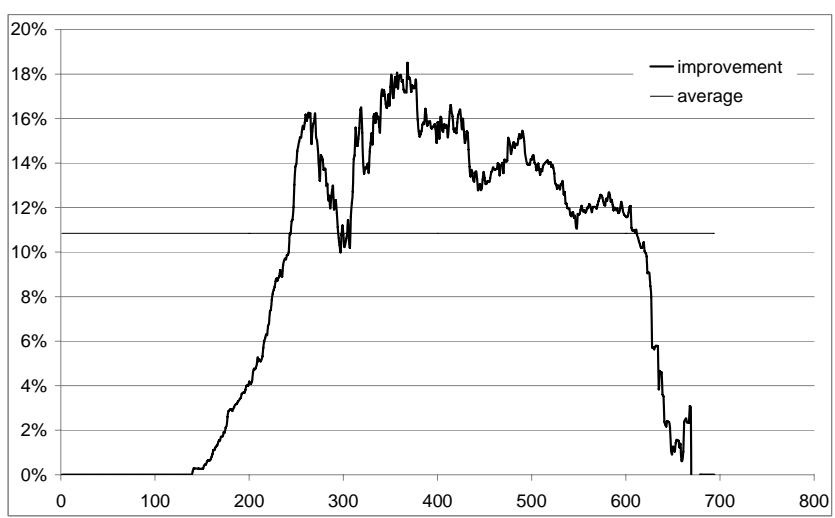

Fig. 8. The improvement of LCMMER compared to the MTPR, in terms of connectivity.

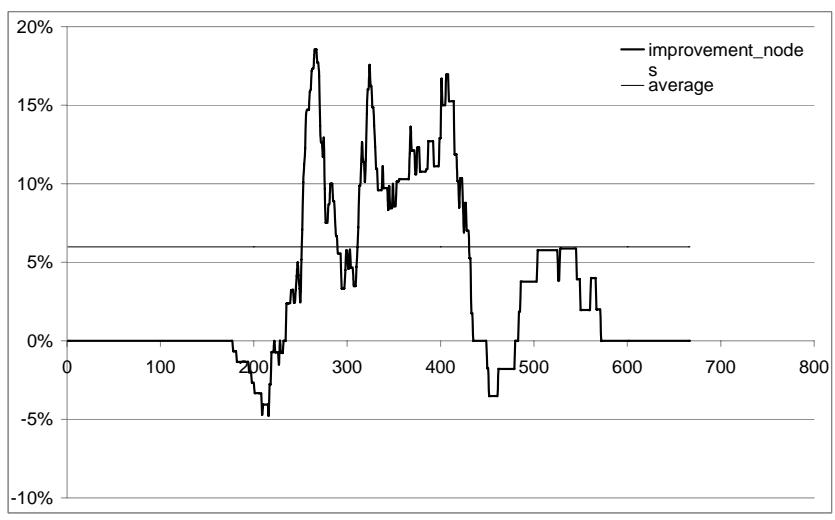

Fig. 9. The improvement of LCMMER compared to the MMBCR, in terms of the number of living nodes.

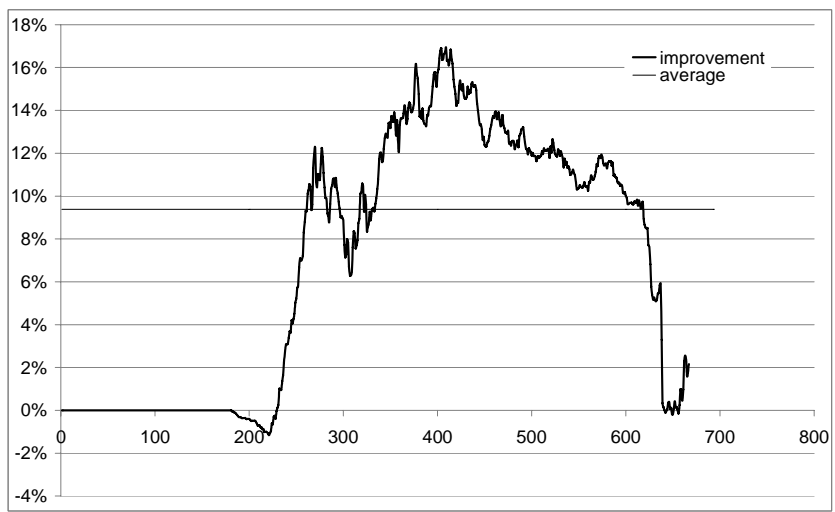

Fig. 10. The improvement of LCMMER compared to the MMBCR, in terms of connectivity.

\section{CONCLUSIONS}

In wireless sensor networks, which are expected to operate unattended for a long period of time, power conservation is a major issue, since it determines the network lifetime. Several 
power conservation schemes have been proposed in the literature for prolonging the lifetime of the sensor network.

This paper presented two routing selection schemes, MTPR and MMBCR, and proposed the new LCMMER scheme. All routing strategies analyzed a set of candidate paths of a specific connection, and determined which one of these paths is used for transferring information from one node to another. The difference between these schemes is the way they select the paths. The MTPR scheme always selects the shortest path from the source to the destination node, and the MMBCR scheme selects the route containing the maximum energy in its least-cost node. The proposed LCMMER scheme selects the route containing the maximum energy in its least-cost node, among all the lowest cost routes. Simulation results showed the comparative advantage of the proposed scheme, over the other two schemes, in terms of network lifetime and connectivity, on a sample topology.

\section{ACKNOWLEDGMENTS}

This work is part of the operational program "Competitiveness", it is funded by the General Secretariat for Research and Technology, Hellenic Ministry of Development and co-funded by the European Social Fund (75\%) and National Resources (25\%), through the PENED 2003 Program - Project "Design and Development Models for QoS Provisioning in Wireless Broadband Networks.

\section{REFERENCES}

[1] Akkaya, K., and Younis, M. A Survey of Routing Protocols in Wireless Sensor Networks. Ad Hoc Network Journal, Vol. 3/3 pp. 325-349, 2005.

[2] Toh C.-K., "Maximum Battery Life Routing to Support Ubiquitous Mobile Computing in Wireless Ad Hoc Networks," IEEE Communications Magazine, June, 2001,pp. 138-147

[3] Al-Karaki, J. N., and Kamal, A. E. Routing Techniques in Wireless Sensor networks: A Survey. IEEE Wireless Communications, December 2004, pp. 6-28.

[4] Yao, Y., and Gehrke, J, “The cougar approach to in-network query processing in sensor networks", in SIGMOD Record, September 2002.

[5] Ye, F., Luo, H., Cheng, J., Lu, S., and Zhang, L. A Two-tier data dissemination model for large-scale wireless sensor networks. In proceedings of the ACM/IEEE MOBICOM, 2002.

[6] Patel, M., Chandrasekaran, R., and Venkatesan, S., "Efficient Minimum-Cost Bandwidth-Constrained Routing in Wireless Sensor Networks", Special Issue on "Wireless Networks and Pervasive Computing”, Journal of Pervasive Computing and Communications (JPCC), Vol 2, No 2, 2006.

[7] Perkins, C. E. Ad Hoc networking. Addison-Wesley, Boston, USA, January, 2001.

[8] Johnson, D. B., Maltz, D.A., Hu, Y.C, Jetcheva, J.G. The Dynamic Source Routing Protocol for Mobile Ad Hoc Networks (DSR). IETF Internet Draft.

[9] Park and Corson, S. Temporally-ordered routing algorithm (TORA) version 1 functional specification. IETF Draft: draftietf-manet-tora-spec-04.txt, 2001.

[10] Perkins, C. E., Belding-Royer, E. M., Das, S. R. Ad hoc OnDemand Distance Vector (AODV) Routing. IETF Internet
Draft, MANET Working Group, draft-ietf-manet-aodv05.txt, March 2000.

[11] Clausen, T., Jacquet, P., Laouiti, A., Muhlethaler, P., Qayyum A. and Viennot L. Optimized Link State Routing Protocol for Mobile Ad Hoc Networks. IEEE INMIC, Pakistan 2001.

[12] Jacquet, P., Muhlethaler, P., and Qayyum, A. Optimized Link State Routing Protocol. IETF Internet Draft, draft-ietf-manetolsr-10.txt, June 2002.

[13] Moy, J. Open Shortest Path First Version 2. RFC 2328, IETF, April 1998.

[14] Haas, Z. J., Pearlman, M. R., Samar, P. The Zone Routing Protocol (ZRP) for Ad Hoc Networks. IETF Internet Draft.

[15] Singh, S., Woo, M., and Raghavendra C. S. Power-aware routing in mobile ad-hoc networks. In Proceedings of the $4^{\text {th }}$ IEEE/ACM International Conference on Mobile Computing and Networking (Mobicom '98), Dallas, TX, 1998, pp. 181190.

[16] Scott, K., and Bambos N. Routing and Channel Assignment for Low Power Transmission in PCS. In Proceedings of the IEEE International Conference on Universal Personal Communications, 1996, pp.498-502.

[17] Cano, J-C., and Kim, D. Investigating Performance of Power-aware Routing Protocols for Mobile Ad Hoc Networks. In Proceedings of the International Mobility and Wireless Access Workshop (MobiWac'02), 2002. 